\title{
Causality and Intervention in the Spin-Echo Experiments ${ }^{*}$
}

\author{
Fernanda SAMANIEGO
}

Received: 29.04.2012

Final version: 15.10 .2012

BIBLID [0495-4548 (2013) 28: 78; pp. 477-497]

DOI: $10.1387 /$ theoria.6136

ABSTRACT: In the so-called "Spin-Echo Experiments" the behaviour of a spin's system seems to violate the second law of thermodynamics. For this reason the "Spin-Echo Experiments" are considered of particular interest for the Foundations of Physics. Interventionists have provided a classical explanation (Blatt 1959; Ridderbos \& Redhead 1998) and a quantum-based explanation (Hemmo \& Shenker 2005) of these experiments. Here both interventionist explanations are assessed by means of the Manipulability Theory of Causal Explanation (Woodward 2003). It is argued that interventionism would gain explanatory depth by providing functional relations and predicting relaxation times.

Keywords: causality; irreversibility; interventionism; explanatory depth.

RESUMEN: En los llamados "experimentos espín-eco" el comportamiento del sistema de espines parece violar la segunda ley de la termodinámica. Por esta razón, los “experimentos espín-eco” son considerados de gran interés en Fundamentos de la Física. Los intervencionistas han ofrecido explicaciones clásicas (Blatt 1959; Ridderbos \& Redhead 1998) y explicaciones basadas en mecánica cuántica (Hemmo \& Shenker 2005) de dichos experimentos. Aquí ambas explicaciones intervencionistas son analizadas mediante la Teoría Manipulabilista de las Explicaciones Causales (Woodward 2003). Se argumenta que el intervencionismo ganaría profundidad explicativa si proporcionara relaciones funcionales y realizara predicciones de los tiempos de relajación.

Palabras clave: causalidad; irreversibilidad; intervencionismo; profundidad explicativa.

\section{Introduction}

The Interventionist approach to Statistical Mechanics, or "interventionism", postulates that the thermodynamic approach to equilibrium is produced by the environmental perturbations acting upon the physical systems. Therefore, if the relevant system of spins were completely isolated in the spin-echo experiments (SE-experiments from now on), interventionism would not be able to account for the behaviour of this particular system. The entropy of the system in the SE-experiments appears to increase and decrease several times violating the second law of thermodynamics. This unique feature of the SE-experiments has generated a fertile ground for debates, not only regarding interventionism, but also regarding the direction of time and the irreversible nature of physical phenomena in general.

* I am very grateful to the members of the research group "Methods of Causal Inference and Representation in Science": Mauricio Suárez, Iñaki San Pedro, Pedro Sánchez, Carlos Corona and Isabel Guerra and also to Orly Shenker, Meir Hemmo, Miklós Rédei, Federica Russo, and María Jiménez Buedo for valuable feedback on previous drafts of this paper. The comments provided by two blind reviewers were also very constructive and helpful. Finally, I would like to thank the Mexican Council of Science and Technology (CONACYT) for the Postdoctoral Fellowship CVU-166476 (2012-2013). 
The objective of this paper is to analyze and compare the classical and the quantum-based interventionist explanations of the SE-experiments. Both explanations are assessed by means of James Woodward's manipulability theory of causal explanation (Woodward 2003).

The manipulability theory is an adequate philosophical framework for the analysis of interventionism for several reasons. Firstly, interventionists explain the SEexperiments by postulating causes and the manipulability theory is a theory of causal explanations. Secondly, the manipulability theory is closely related to controlling causal factors and this perspective fits very well with the experimental context of the SEexperiments. Thus the manipulability theory provides us with a tool suitable to evaluate and compare different causal explanations of these experiments. Moreover, the concept of intervention itself is prominent and central to the manipulability theory.

The analysis presented in this paper is relevant for understanding in detail the irreversible process that takes place during the SE-experiments; it clarifies the difficulties that a satisfactory version of the interventionist approach must overcome; and, additionally, some interesting features of the manipulability theory are revealed.

The content of this paper is organized as follows. The philosophical and methodological framework, namely, the manipulability theory, is presented in section 2 . In section 3, statistical mechanical interventionism is introduced and the SE-experiments are described in detail. Three philosophically relevant questions about the experiments are then answered from the interventionist perspective. Section 4 develops the full analysis of the interventionist explanations of the spin-echo experiments in terms of the manipulability theory. This is the main original contribution of the paper since the manipulability theory has never been applied to statistical mechanical interventionism before. The results of the analysis are discussed in section 5. And finally, section 6 contains the overall conclusions.

\section{The Manipulability Theory of Causal Explanation}

The basic idea of the manipulability theory is that causal explanations offered in natural and social sciences, do not aim simply at satisfying our intellectual curiosity, but are often guided by the goal of manipulating and controlling the explained events. Accordingly, the essence of a causal explanation in the manipulability theory consists in "exhibiting a pattern of counterfactual dependence between explanans and explanandum - a pattern of counterfactual dependence of the special sort associated with relationships that are potentially exploitable for purposes of manipulation and control" (Woodward 2003, 13, 16).

In satisfactory causal explanations, the patterns of dependence between causes and effects are invariant under a set of testing interventions. This claim contains the main notions of the manipulability theory. As a general illustration of these notions let us consider a string with a weight hanging on it. Suppose that a putative causal explanation, based on Hook's law, affirms that the vertical force-exerted by the weight-causes the elongation of the string. According to the manipulability theory, we can test this causal explanation by manipulating or intervening on the values of the putative cause and studying the changes in the values of the putative effect. In this particular case, an intervention 
may consist in hanging different weights exerting forces of different magnitudes upon the string. If Hook's law correctly predicts the values of the elongations under this particular intervention (i.e., if Hook's law is invariant, holds, under this particular intervention) then the explanation has some explanatory import.

\subsection{Notion of Cause}

- $C$ is a genuine cause of $E$ if, given the appropriate background conditions, there is a possible manipulation of the cause $C$ such that this is also a way of manipulating or changing the effect E. (Woodward 2003, 16)

\subsection{Notion of Intervention}

Interventions are changes on the putative cause $C$ performed in such a way that if any change occurs in the effect $E$, it occurs only in virtue of $E$ 's relationship to $C$ and not in any other way. An intervention $I$ must meet the following conditions (Woodward 2008, section 5; Woodward 2003, 98):

$I N-i$ The intervention $I$ completely disrupts the causal relationship between $C$ and its previous causes. The value of $C$ is set entirely by $I$.

$I N-i i$ The intervention $I$ should not itself be produced by any process that affects $\mathrm{E}$ via a route that does not go through $C$.

IN-iii The intervention $I$ leaves the values taken by any causes of $\mathrm{E}$ except those that are on the path from $I$ to $C$ to $E$ unchanged.

$I N-i v$ The intervention $I$ must not directly cause $\mathrm{E}$ via a route that does not go through $C$.

\subsection{Criterion of Explanatory Depth}

- A causal explanation is explanatorily deep if the causal relationships figuring in the explanation remain invariant under a wide and diverse range of interventions (Woorward 2003, 211-215, 311)

If a given explanation meets this criterion (2.3) of explanatory depth, then it is relevant to the manipulation and control of the explained event. Therefore, according to the manipulability theory, this is what any causal explanation should aim for.

\section{Interventionism and the Spin-echo Experiments}

\subsection{Distinctive features of interventionism}

The approach to statistical mechanics called interventionism intends to explain the irreversible behavior of thermodynamic processes. According to interventionism, the random influence of the external environment acts as a source of perturbation over the system. And it is precisely this environmental disturbance that causes the irreversible increment of entropy. Hence, the essential idea posit by interventionism is that real systems are never isolated from their surrounding environment; On the contrary, they 
are in constant interaction with it. Isolation, if possible, is only achievable for finite and very short times. For any larger time range, for most physical systems, the interaction with the environment becomes crucial.

The origins of interventionism can be traced back to the mid 20th century, more precisely, to Peter Bergmann and Joel Lebowitz's paper 'New Approach to Nonequilibrium Processes' (1955) and to Reichenbach's book The Direction of Time (1956). Also the model that J.M. Blatt put forward a few years later (1959) is one of the pioneer interventionist proposals. These early interventionists of the 1950's were followed some decades later by M. Redhead and T.M. Ridderbos (1998). From now on we will refer to all these authors as "the classical interventionists". More recently, Meir Hemmo and Orly Shenker (2003 and 2005) have put forward an explanation of irreversibility based on quantum decoherence for open systems. Despite the fact that Hemmo and Shenker's approach importantly differs from the classical interventionist efforts in the sense that it appeals to quantum mechanics, the authors name themselves interventionists because the interaction between the system and the environment plays a crucial role in their explanation of the approach to equilibrium. To expand on the interventionist position we need first consider the SE-experiments. In the next section (3.2) we describe the experiments in detail; and in sections 3.3 and 3.4 we explain the interventionist models of the SE-experiments.

\subsection{The Spin-Echo Experiments}

In the SE-experiments a system of spins suffers several changes due to magnetic alterations. During the experiment it is actually possible to control some of the microscopic variables of the system-something usually impossible for thermodynamic systems. And secondly, isolation from external influence is highly controlled. This combination of features is rarely found in a single experimental device. Additionally, the SEexperiments represent a challenge particularly for interventionists because the system of spins fluctuates from a state of high entropy to a state of low entropy (and vice versa) and the environment seems to play no role at all during the whole process.

The underlying mechanism of the SE-experiments can be understood using an analogy offered by Erwin Hahn (Hahn 1953, 5) who first carried out the experiment in the 1950's. The analogy consists of an imaginary Olympic race. The relevant system is the group of runners in this race. The runners are aligned along the starting line marked on the track (a very ordered state). The race takes place and when it finishes some runners are ahead of others so the positions of the runners are not longer aligned (representing a disordered state of the system). Now let us imagine that the runners are standing in their final positions and we ask them to turn around and start running back. Assuming (for the sake of the adequacy of the metaphor) that every runner will equal the velocity she had during the first phase of the race, the runners will reach their original positions at the starting line and recover the ordered alignment they had at the start of the race. If the duration of the original race is $\tau$ the runners will regain their original positions after a total time of $2 \tau$.

In a SE-experiment we have a set of nuclear spins (normally belonging to protons in a sample of glycerine) placed in a strong magnetic field. Through the application of 
a first radio-frequency pulse, the spins are initially aligned. In other words, the initial positions of the spins configure an ordered initial state. Let us say, for example, that the direction of the magnetic field is in the z-axis while the superposed spins lie over the xy-plane and they are all pointing in the same direction (as shown in Fig. 1 below). This produces the emission of a macroscopic electromagnetic signal.

The spins are then left to evolve for a while. In a perfectly homogeneous magnetic field all the spins should precess clockwise with the same frequency, as a single clock hand. However, the discontinuities in the magnetic field cause slight differences in the precession rates of the spins (see Fig. 2 below). The precession rate of each spin is more or less affected depending on the strength of the field at each point. The evolution of the spins' system during this stage is analogous to the first 'race' among the runners. After an interval of time $(\tau)$, the spins reach a disordered state, in which they are not pointing in the same direction anymore (as in Fig. 2 below) and the macroscopic electromagnetic macroscopic signal completely disappears.

In a second part of the experiment (which corresponds to the runners running back) another radio-frequency pulse is applied rotating $180^{\circ}$ the xy-lane as shown in Fig. 3 and Fig. 4 below.

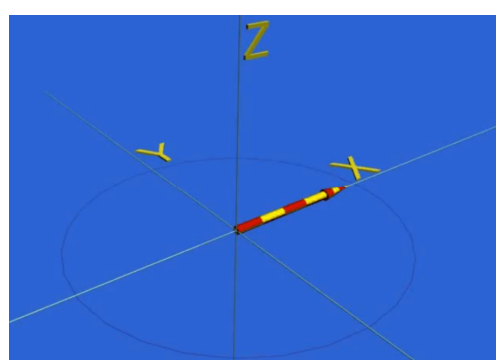

Figure 1

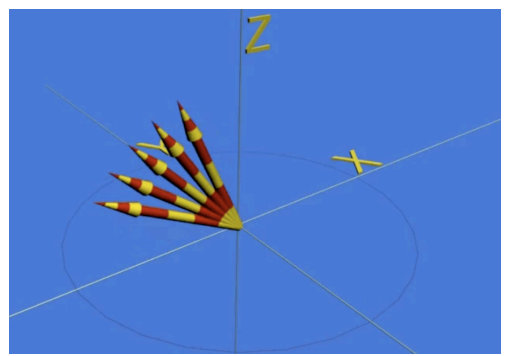

Figure 3

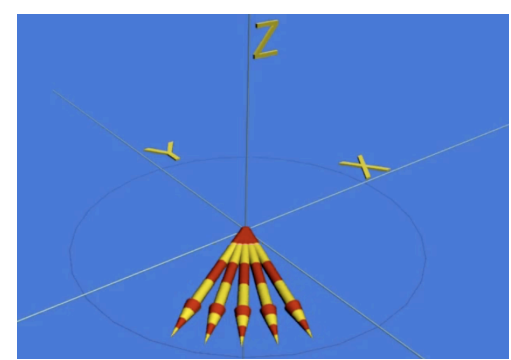

Figure 2

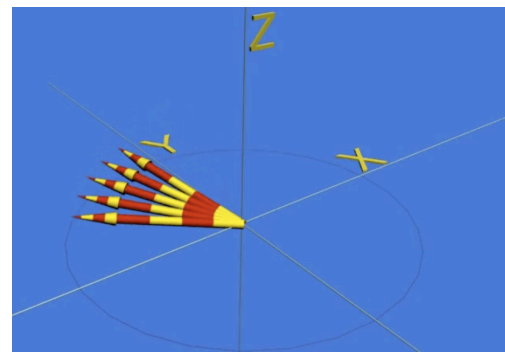

Figure 4

The spins are then left to evolve freely again so they continue precessing clockwise. After an interval of time $(2 \tau)$ the spins return to an ordered state equal to its original state (shown in Fig. 1). And this causes the re-emission of the macroscopic electro- 
magnetic signal. This repetition of the signal is the phenomenon that gives the name to the experiments.

We may repeat the procedure again on the system any number of times. We can control the radio-frequency pulses in such a way that we successively produce and destroy the alignment of the spins. As a result, every time that the spins are in phase the electromagnetic signal is emitted; and every time the spins are out of phase the signal disappears. So we have here a succession of signals in time. However, not all the echo signals are identical. Precisely as in an echo, the maximum intensity of the electromagnetic signals always decreases until the signal eventually disappears (see Fig. 5 below). And, at some point in the system's evolution, more precisely, after an interval of time known as relaxation time, the system is unable to generate the echo-signal.

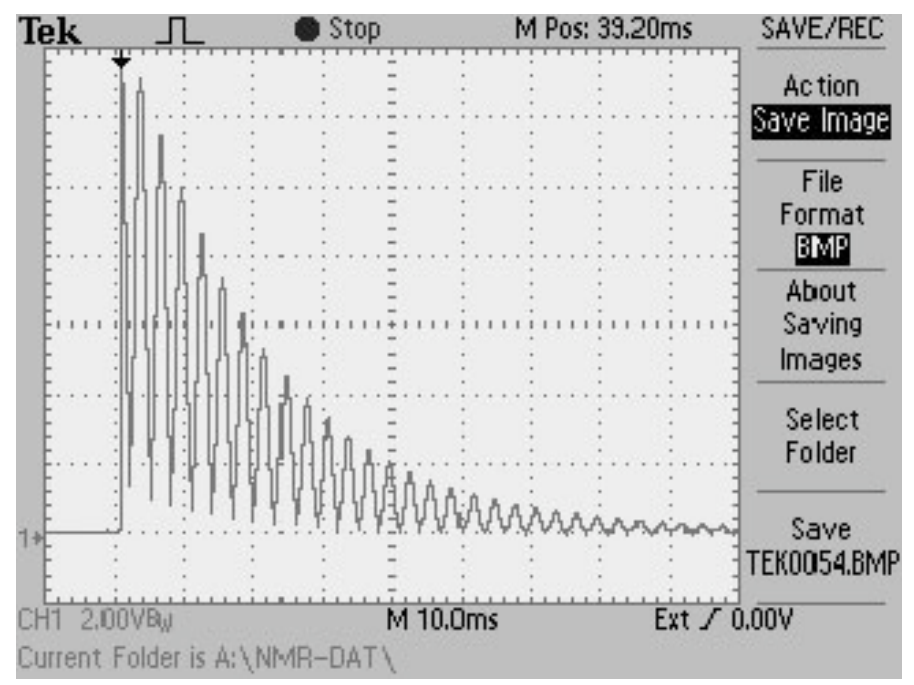

Figure 5: Intensity of the signal in the spectrometer

To summarize, three relevant questions emerge from the SE-experiments:

Q1: Is the second law of thermodynamics violated in a SE-experiment?

Q2: How can we explain the decay and disappearance of the echo signal in successive repetitions of the radio-frequency pulses?

Q3: How do interventionist approaches account for the evolution of the spin's system?

\subsection{Classical Interventionism and the SE-experiments}

As regards question $Q 1$ interventionists believe that the SE-experiments do not violate the second law of thermodynamics. Let us assume that the violation of the second law requires a decrease of entropy to occur in a closed system evolving spontaneously. Then, in order to deny that the second law of thermodynamics is violated in the SE-experiments, interventionists may follow three different strategies (or combina- 
tions thereof): They can deny that there is entropy decrease during the SE-experiments; Alternatively, they can argue that in the SE-experiments the system of spins is not closed; Or, finally, they can argue that the evolution of the spin's system in the SE-experiments is not spontaneous but controlled. Let us consider these three strategies in turn.

\subsubsection{Entropy in the spin-echo experiments}

The first strategy has been adopted, for instance, by Blatt (1959) and Ridderbos and Redhead (1998). These classical interventionists argue that, although from the macroscopic point of view the system of spins seems to reach a state of equilibrium when the spins are out of phase, in fact the correlational information about the initial order is still contained in the system. That is the reason why the system of spins is still able to recover its initial order. Such a state, in which the spins 'remember' the information about their initial state, corresponds to what Blatt $(1959,749)$ called a state of quasiequilibrium. However, the state of true equilibrium is only reached when the correlational information has been dissipated and, once a system arrives to such a state of true equilibrium it stays there forever. Once the system is in this state of true equilibrium, the initial state of the system cannot be restored anymore because the correlational information has vanished on the microscopic level. (Ridderbos \& Redhead 1998, 1256). In other words, when a system under study is in a quasi-equilibrium state the order has disappeared at the macroscopical level, but it is still 'hidden' at the microscopic level in the form of correlational information. In states of true equilibrium, by contrast, there is no such a 'hidden order'. If the nuclear spins were in a state of true equilibrium, they would not be able to get back in phase again.

The classical interventionists' argument is applied to the case of the SEexperiments as follows. The system of spins neither evolves toward true equilibrium after the first radio frequency pulse, nor does it evolve away from true equilibrium after the second $r-f$ pulse. In the interval of time $[0,2 \tau]$ the system of spins only fluctuates from states closer or farther to quasi-equilibrium. The relevant question is: how the usual descriptions of entropy in SM should be modified or replaced in order to correctly describe the thermodynamically normal evolution of the system of spins in the SE-experiments? In other words, according to classical interventionists, the concept of entropy itself must be re-defined, in such a way that this new entropy never decreases in the SE-experiments, and therefore the second law of thermodynamics is never violated.

\subsubsection{The system of spins is open}

The second strategy consists in denying that the system of spins is closed during a SEexperiment. Form this perspective the system of spins interacts with the environment. Due to this interaction some energy of the system is dissipated. And the energy dissipation causes the decay of the echo signal (more details bellow).

Since this strategy offers an explanation of the echo signal decay, it provides an answer to question $Q^{2}$. As a matter of fact, the intensity of the echo signal decreases and 
eventually disappears, even if the radio frequency pulses are still repeatedly applied on the spin-echo system. Interventionism can explain this fact by appealing to the interaction between the system of nuclear spins and its environment. This is an advantage of interventionism compared with other approaches to the irreversibility problem that fail to explain this aspect of the spin-echo experiments.

Let us see how exactly the system of spins is said to interact with the environment. We are hence looking for a progressive dissipation effect in the SE-experiments that, in terms of the race analogy, help us explain why not all the runners went back to the starting line (or at least not as fast as they had run in the original race). Then, what we need is a physical process that makes spins 'lose their memory of their initial state', or some kind of energy dissipation that affects the spin's frequency of precession, preventing them from regaining the spin-alignment. Magnetic energy is exchanged during the experiment in several different ways. Sometimes the nuclear spins transfer their magnetic energy of precession to the sample molecules in the form of kinetic energy. The magnetic energy of precession is some other times transferred to the neighbouring spins.

Additionally, the system is affected by the Brownian motion of the glycerine molecules and the fluctuations in the local magnetic fields due to neighbouring moments. These two latter phenomena may drive the momentum of some molecules from the static magnetic field (chosen by the experimenter and controlled with the radio frequency pulses) into another randomly differing magnetic field (Hahn 1953, 6). As a consequence, the spins' frequency of precession is perturbed.

The presence of these effects is used by classical interventionists to defend the view that, during the SE-experiments, the spins change their frequency, not only as a result of their interaction with the experimental set up but also because they transmit magnetic energy to the environment. The spins that relax their energy and are not in phase with the rest of the spins will not contribute to the next echo-signal. Due to this constant disturbance from the environment, the number of out-of-phase spins increases during the experiment and this explains why the intensity of the echo-signals is progressively reduced.

In Blatt's terminology, completely isolated systems never reach true equilibrium, instead they always stay in a state of quasi-equilibrium. In this state the velocities of the system can still be reverted showing that the system 'remembers' the information about its initial state. This is the case for the system of spins at $t=\tau$ (i.e. when the spins are out of phase for the first time). However, once the $r-f$ pulses are applied many times the echo-signal eventually disappears. The system of spins has then reached a state of true equilibrium and the process is not longer reversible. According to classical interventionists, the gradual decay in the intensity of the echo-signal proofs that the correlational information dissipates into the environment, and this only makes sense if we conceive the system of spins as an open system.

Let us sum up the evolution of fine-grained entropy during a SE-experiment. In the first part of the experiment the effect of the environment is almost imperceptible and entropy (more precisely, fine-grained entropy) is conserved. In the second part of 
the experiment, and during the consecutive echo-signals, the environment increasingly affects the system, diffusing its correlational information.

\subsubsection{Controlled versus free or spontaneous evolutions}

So far, we have said nothing about the third strategy to deny the violation of the second law of thermodynamics. This strategy consists in denying that the evolution away from equilibrium manifested in the spin-echo experiments is free or spontaneous. In other words, it is argued that the second law only applies to closed systems that evolve "on their own", i.e., free of external influences. A decrease of entropy in a system evolving under such circumstances would genuinely represent a violation of the second law. However, the evolution of the system of spins in a SE-experiment, far from spontaneous, may be rather considered as a highly controlled evolution.

This strategy, however, is problematic in the sense that classifying evolutions as 'controlled' or 'spontaneous' seems to depend on our election of the relevant system under study (see Shenker 2001). For example, if in the SE-experiments we only take the system to be the set of nuclear spins, it seems that the evolution has been induced and controlled from the outside world (via the pulses). By contrast, if the environment is taken to be a part of the system itself the evolution seems spontaneous.

We have so far explicitly addressed questions $Q^{1}$ and $Q^{2}$. However, question $Q^{3}$ has been addressed implicitly too in the previous sections. As mentioned, classical interventionism accounts for the SE-experiments by appealing to the distinction between quasi and true equilibrium and rejecting Gibb's definition of coarse-grained entropy. We may turn now to a discussion of the quantum based interventionism, and how that approach explains the evolution of the spin-echo system.

\subsection{Quantum-based interventionism}

Meir Hemmo and Orly Shenker $(2003,2005)$ proposed an explanation of the irreversible thermodynamic behaviour based on the underlying quantum mechanical dynamics. More specifically, Hemmo and Shenker appeal to quantum mechanical models of environmental decoherence to account for the interaction between the system and its environment at the quantum level. This interaction, combined with the stochastic nature of the quantum dynamics, leads to the irreversible increase of entropy. According to Hemmo and Shenker, quantum decoherence is the mechanism that "brings about an approach to equilibrium in the classical sense of, for example, an evolution towards the most probable macrostate" (Hemmo \& Shenker 2003, 348).

In the stochastic no-collapse interpretations of quantum mechanics some additional dynamical laws are introduced in the quantum description of the system. These laws are said to produce the so-called effective collapses, which, in contrast with the usual collapses of the quantum state, are not considered as real collapses. Hemmo and Shenker built their answer to the irreversibility problem on such no-collapse interpretations of quantum mechanics, assuming that "when macro-systems undergo decoherence interactions with their environment the extra dynamics results in effective collapses onto 
coherent states corresponding to what [the authors] have called quantum mechanically normal states" (Hemmo \& Shenker 2005, 632).

From this perspective, the total quantum state evolves in accordance with the Schrödinger equation. Due to decoherence, effective collapses of the quantum state will bring about transitions from one effective state to another one. In other words, the system seems to jump from one Schrödinger trajectory to another one. This is the so-called effective collapse. A system's effective state is not uniquely determined by its previous effective state. On the contrary, "transitions between effective states are genuinely stochastic [and] on this assumption the result is that the effective state of the thermodynamic system changes in a stochastic way in the course of decoherence" (Hemmo \& Shenker 2003, 351). In this way, the stochastic nature of the system's evolution, according to this approach, is the result of decoherence together with the stochastic extra dynamic laws.

In order to recover the predictions of classical statistical mechanics, Hemmo and Shenker postulate the following dynamical hypothesis: "The quantum mechanical probabilities [produced by the extra dynamical laws] reproduce the quantitative predictions of classical statistical mechanics" (Hemmo \& Shenker 2003, 633) Although the authors recognize that they cannot provide a proof of their dynamical hypothesis, they argue that its plausibility can be defended on the basis of the characteristics of spontaneous effective collapses. More precisely, they argue that effective collapses are extremely frequent in macroscopic systems under decoherence. And these collapses induce extremely small changes in position comparable with changes in position at statistical mechanical scales.

In sum, the irreversibility problem is solved in the decoherence-approach by offering a mechanism, viz., effective collapses in decoherence situations, which guarantee that the evolution of a macroscopic system has a high probability of being thermodynamically 'normal'. Let us see now how this applies specifically to the case of the spinecho experiments.

According to decoherence-based approach, the system is not only affected by the external interaction with the environment (as in classical interventionist approaches) but it is also affected internally by the stochastic dynamics, i.e., by the effective collapses. This is also manifested in the double role played by decoherence in the SE-experiments: On the one hand decoherence directly affects the spins through the influence of the environment. On the other hand, a diffusing effect is generated by the interaction between spins themselves, which influences their states. Let us call these decoherence processes external and internal decoherence respectively.

The internal decoherence, i.e. the interaction between spins, leads in turn to the echo-signal decay by a process that possesses two different stages. In a first stage the spin-spin interaction induces stochastic effective collapses. In the second stage, each collapse of a spin produces a kind of "holistic" diffusing effect in the spin states of other particles. A direct consequence of this is that, if a decoherence based approach is correct, an experiment in which the spin-spin interaction is reduced (for example, by diluting the sample in the glycerine) should slow-down the decay of signal intensity. 
This opens the way to possible experimental tests for the decoherence-based approach to the problem of irreversibility.

\section{Test of Explanatory Depth}

In this section we examine how deep the interventionists' explanations are in the Woodwardian sense of explanatory depth (defined in section 2.3). In section 4.1 we analyse whether the causal patterns postulated by classical interventionism remain invariant under some interventions. In section 4.2 we apply the same "manipulability test" to the causal patterns postulated by quantum-based interventionism.

\subsection{Testing the classical interventionist explanation}

We will first propose some actual or hypothetical manipulations to control the values of the cause(s) postulated by each explanation. Afterwards, we will verify which of them fulfil Woodward's conditions INi-iv (defined in sec.2.2). This will provide us with a set of interventions for testing the explanations under study. We will then proceed to analyse if the causal links postulated by each explanation remain invariant when such interventions are applied.

Our description of the classical interventionist explanation in manipulability theory terms (illustrated in Fig. 6 below) assumes that the interaction between the environmental perturbations exerted upon the system of spins are brought about by the thermal interactions between the spins and the lattice, the magnetic fluctuations and the Brownian motion. Let us examine how exactly those factors increase or decrease the rate of environmental perturbation. Expressed in the manipulability theory terms, let us study how these three putative interventions can be used for manipulating the value of the causal variable EP (environmental perturbations) with respect to the effect variable $H$ (height of the echo-signal).

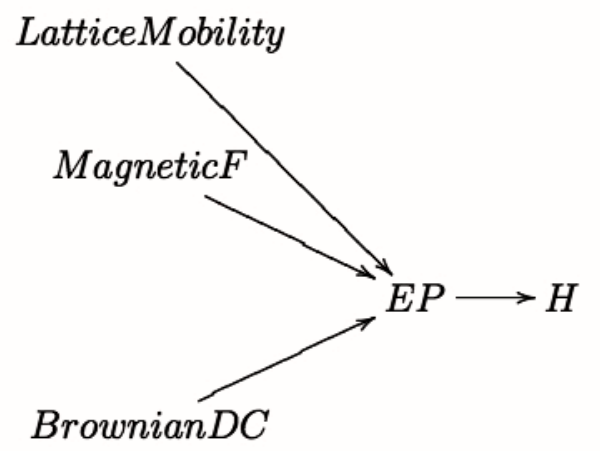

Figure 6: Directed graph for classical interventionist explanation ${ }^{1}$

\footnotetext{
${ }^{1}$ As one reviewer correctly points out, "lattice mobility", "magnetic fluctuations" and "Brownian motion" could be thought as constituents of EP, rather than as causes of EP. And this would lead us to a completely different analysis. However, Fig. 6 follows as literally as possible classical interventionists,
} 
In order to intervene upon the variable $E P$, we need to propose ways of wiggling the values the three putative intervention variables showed in Fig. 6. Let us begin with the Brownian diffusion coefficient, which is frequently recognized in scientific papers as a relevant factor affecting the behaviour of the spin-echo system. As Einstein pointed out in one of his annus mirabilis papers, the Brownian motion depends both on the temperature and viscosity of the substance in which the particles are immersed. Thus in the SE-experiments the value of the Brownian diffusion coefficient may be manipulated, for example, by diluting the glycerine in water; or by warming the sample up.

An experiment comparing the spin-echo signal for different dilutions of water and glycerine has been recently performed (Martin \& Hughes 2006). The results of this experiment show that the relaxation time of the system actually depends on the waterglycerine proportion in the sample. More precisely, the higher the glycerine content, the shorter the relaxation time. These results are consistent with the results obtained by Bloembergen, Purcell and Pound (1984) in a similar experiment.

Classical interventionism would account for this behaviour by arguing that the Brownian motion increases when the concentration of glycerine is high. So the perturbations are intensified making the echo-signal disappear (i.e. bringing the system to a state of true equilibrium) in a shorter relaxation time.

The predictions provided by classical interventionism are compatible with the experimental fact that variations in relaxation time have been seen to depend on the specific Brownian motion rate associated to the actual viscosity and temperature of the sample. This is the kind of manipulation we are looking for.

Let us now consider manipulations through variations of the magnetic fluctuations. Perhaps the rate of magnetic fluctuations can be raised during the experiment by introducing some paramagnetic impurities in the sample, or by moving some extra magnets around the experimental device. This would generate magnetic fields in certain areas of the sample, additional to the static magnetic field generated by the original device. In the only experiment I have been able to find performing this kind of manipulation (Stejskal 1964) a technique is employed for generating magnetic gradients with a couple of coils wound on tapered forms. The effect on the system is then measured for several different gradients. The output of this experiment shows that the echo-signal is directly affected by the magnetic gradients in such a way that greater magnetic gradients correspond to echo-signals with weaker intensities.

An alternative would be to prevent (rather than generate) magnetic fluctuations and in-homogeneities. Some experiments have already been performed in order to achieve this. Bloembergen et al, for example, describe how water samples can be used to detect the field in-homogeneities and find, in that way, the more homogeneous area in the field (see Bloembergen et al 1984, 684). Also, and more recently, a computer model has been developed to simulate different in-homogeneous magnetic fields and study how much the behaviour of the spin's system is affected by these inhomogenei-

who always quote Hahn on this. And Hahn explicitly claims that EP is "brought about" by these factors (see, for example, Hahn1950, 581; or Hahn 1953, 7). 
ties (Nyenhuis 1994). In order to reduce costs and improve the medical application of the spin-echo technique, the computer model aims to find the range at which the measurements of relaxation times are still reliable without using too expensive magnets. For our purposes, it is enough to realize that there are some experimental manipulations that effectively test the putative influence of the internal magnetic fields upon the spin-echo system.

It is also worth mentioning that immersing the sample in a perfectly homogeneous magnetic field is also a hypothetically conceivable manipulation; therefore, it may count (if it fulfils conditions $I N i-i v$ ) as an intervention for testing our explanation under study.

Again, classical interventionists would be able to account for the behaviour of the system under variations (increases or decreases) of the amount of magnetic field fluctuations. More precisely, they would predict longer relaxation times for the perfectly homogeneous field, and shorter relaxation times for fields with more inhomogeneities and magnetic fluctuations.

Let us finally imagine how the thermal interactions between the spins and the lattice can be modified. As Hahn explained already as back as 1950, the spin-lattice thermal interaction brings about transferences in the spins of some particles (from spin up to spin down). When this occurs the magnetic energy of the precessing spin is transferred to a molecule of glycerine in the form of kinetic energy. After many spin transferences the system experiences a "cooling process" characterized in practice by the so-called spin-lattice relaxation time.

These random thermal interactions between the spins and the lattice are present during the whole experiment. They produce the de-phasing of the spins in the short term (driving the system to the state that classical interventionists called quasiequilibrium), and the decay of the eco intensity $\mathrm{H}$ in the long term. According to the classical interventionist explanation, this is the dominant process affecting the spin frequencies. From their point of view, in the absence of the spin-lattice thermal interaction, the height of the echo-signal $H$ would simply never decrease (see Ridderbos \& Redhead 1998, 1252-1253).

The relevant question for us is whether we can use this spin-lattice interaction to intervene on EP, i.e., to control the values of the putative causal variable EP (environmental perturbations). Intervening to stop the environmental perturbations would imply isolating the spins from the molecules to which they belong. Is this even conceivable?

From the classical interventionist perspective, all the systems in the universe are open, except the universe as a whole. But this is a contingent matter of fact, and not a necessary matter of law. Recall that, according to the manipulability theory it is not necessary to actually perform the intervention in order to consider it valid. When technological limitations are involved, we invoke hypothetical interventions to analyse the causal relationships. The only requirement is that such intervention be physically possible (see Woodward 2003, section 3.5). If we were studying some other system, a box full of gas for example, "absolutely isolating the system from its environment" would imply blocking the interaction between the gas molecules and the walls of the 
box. And this, perhaps, would count as a possible hypothetical manipulation. However, in the spin-echo experiments, isolating the system implies a change in the very capacity of the spins to transfer their energy to the molecules in the sample. Can we genuinely modify this? I can hardly imagine such a manipulation, unless we replace the glycerine with some other substance (or other tissues as a brain or a heart tissue) whose molecules produce different splits between spin up and spin down.

Changes in the molecular structure of the sample change the capacity of spins to flip from one state to the other; and hence the spin-lattice thermal interaction is also affected. In other words, the lattice mobility depends on the molecular structure of the sample. But, even if we manage to reduce the spin-lattice interaction, that interaction will never be completely blocked. So let us assume that there is no way of isolating the spins from the lattice and continue with our analysis.

Summing up, we have so far proposed four different manipulations for controlling the values of the variable $E P$, which is the putative cause of irreversibility according to classical interventionism:

- Reducing the viscosity of the sample (M1).

- Changing the temperature of the sample as $(\mathrm{M} 2)$.

- Introducing magnetic in-homogeneities (M3); and

- Producing a perfectly homogeneous field (M4) (hypothetical manipulation)

Let us now consider which among them fulfil the conditions $I N i$-iv (defined in section 2.2). If these manipulations fulfil the conditions $I N i$-iv they will count as testing interventions. If the postulated causal relations by classical interventionism (in Fig. 6) turn out be invariant under such testing interventions, we will be able to determine if the classical interventionist explanation meets criterion of explanatory depth (see 2.3).

In order to count as a testing intervention, the manipulation M1 should first fulfil the condition $I N-i$, i.e. M1 should switch off the effect of any alternative cause(s) of the intervened variable. Thus, in order to fulfil the condition in this particular case, the manipulation M1 (diluting the sample) should switch off the effect of the magnetic fluctuations upon the spin's frequencies and, additionally, block the spin-lattice energetic exchange.

It has been found in practice, however, that both "magnetic fluctuations" and "spin-lattice thermal interactions" are still acting when the manipulation $\mathrm{M}_{1}$ is carried out. Moreover, it is impossible to conceive any manipulation that controls the Brownian motion, and, at the same time, neutralises the exchange of magnetic energy between spins. Therefore, M1 does not fulfil $I N-i$. And thus, despite the fact that $\mathrm{M}_{1}$ meets other conditions on interventions, it cannot be considered as a testing intervention. The second manipulation $\mathrm{M}_{2}$ also fails to meet one condition. The evolution of the spin-system is temperature-dependent. So, cooling or warming the sample up, violates the condition $I N-i i$, according to which the intervention cannot be a direct cause of the explained event. In other words, the intervention must affect the effect only trough the path containing the postulated cause.

The third and fourth manipulations $\mathrm{M}_{3}$ and $\mathrm{M}_{4}$ fail to meet condition $I N-i$. The reason is analogous to the reason why M1 does not fulfil the condition. But in this 
case the violation of $I N-i$ is due to the impossibility of "switching off" the effect of the Brownian motion upon the spins frequencies, and the spin-lattice thermal interaction, while the magnetic manipulations are performed.

Our conclusion in this section (4.1) could be the following: All the processes taking place during the spin-echo experiments to which classical interventionists assign a causal role (namely, magnetic fluctuations, Brownian motion and thermal interactions) are closely related to each other. The causal structure seems to be complex and no intervention is a clean single direct cause upon the putative cause. As a consequence, all the manipulations ( $\mathrm{M}_{1}$ to $\left.\mathrm{M}_{4}\right)$ violate at least one of the conditions $I N i$-iv. So we are left with no interventions to test the putative causal relationships postulated by classical interventionism.

\subsection{Testing the decoherence-explanation}

Our second explanation under study, the decoherence-based explanation is illustrated in Fig. 7 (below). The internal and the external decoherence are represented by variables DI and DE respectively; EC stands for "effective collapses" and $H$ stands for "height of the echo signal". In order to analyze it in terms of the manipulability theory, let us propose some manipulations designed to intervene the values of the putative causal variables $D I$ and $D E$, with respect to the effect variable $H$ (height of the echosignal).

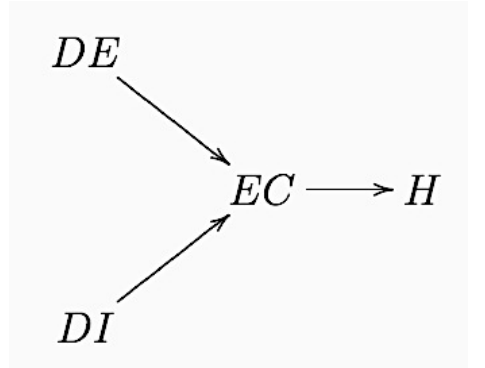

Figure 7: Directed graph for the quantum-based interventionist explanation

The manipulation M1, consisting in diluting the glycerine in water, is again a good candidate. If we add water to the sample, the distance between the glycerine molecules increases and each spin gets away from the others. Hence, if the decoherence approach is correct, decoherence rate decreases, thus slowing down the system's approach to equilibrium.

Is there any other method for reducing decoherence? And furthermore, is it possible to completely avoid it? Answering these questions has become one of the main goals of researchers in quantum information (Uhring 2009). Reducing decoherence enables us to keep quantum information for longer times. And completely avoiding decoherence would be idyllic for building the so-called quantum computer. For these reasons, and independently of its philosophical relevance for the foundations of statistical mechanics, suppressing decoherence in the spin-echo experiments has recently become a research field of great interest. As a result, during the last few years, several 
different sequences of radio-frequency pulses have been put forward seeking to reduce decoherence as much as possible or for as long as possible (see for example Uhring 2007 and 2009).

Similar multi-pulse techniques had been proposed much earlier (1950's) by Carr and Purcell (1954) and soon after improved by Meiboom and Gill (1958). However, these techniques were interpreted in previous decades as ways of improving the resolution and precision of the experiments. And they were focused on getting better information about the chemical composition of a given sample. In fact, the so-called Carr-Purcell-Meiboon-Gill sequence of pulses has been widely applied in medicine for producing images of body structures in order to detect brain tumors, osteoporosis, heart diseases, etc.

The novelty of more recent work (for example Uhring 2007 and 2009) is that the improved technique for "dynamic decoupling" is explicitly proposed as a way of suppressing decoherence.

In terms of the runner's metaphor (explained in section 3.2), the essence of the multi-pulse techniques consists in "not letting the racers run too far". Imagine that all the runners are placed in the starting line. The racers begin to run at different velocities but before they lose the alignment (perhaps just an instant after the race begins) we stop them and ask them to run back. Again we allow them to run only a small distance. And soon multiple races are conducted. As a consequence, the original distribution remains almost unchanged.

This situation has been experimentally developed with spins obtaining successful results. The improved sequences of $\mathrm{r}-\mathrm{f}$ pulses bring about less diffused spin-echo patterns in which the spins' system reaches the equilibrium in a longer relaxation time. In other words, the decay of the signal is softer, the diffusion is largely circumvented and the spins are able to keep the phase-memory for much longer $(2$ seconds instead of 0.2 seconds).

In the latest models (Uhring 2007 and 2009) the optimized sequence of pulses is said to suppress decoherence even more efficiently than the so far known sequences of equidistant pulses. Two additional advantages have been supposedly achieved. First, the results are less sensitive to thermal effects. And, second, the number of pulses required to get a certain prolongation of the relaxation time can be much smaller (see Uhring 2009, 100504-4).

As mentioned above, researchers in quantum information aim to find the way to avoid decoherence for longer times. In fact it would be ideal for them to completely avoid it. For our analysis in terms of the manipulability theory it would be also very convenient to find a manipulation such that decoherence does not take place during the whole experiment. In such a situation, according to Hemmo and Shenker's decoherence-based explanation, the system should never approach equilibrium. One way of doing so is setting up the system in a "no-decoherent quantum state". This kind of state has not been prepared and, as at present, it is not considered an experimental possibility yet. However, it remains a theoretically conceivable quantum state, and thus counts as a manipulation in our analysis. 
In sum, we have proposed the following prima facie possible manipulations for testing the decoherence-based explanation:

- Reducing the viscosity of the sample (already labelled as M1);

- Reducing decoherence through multi-pulse sequences (M5);

- The "no-decoherent" quantum state (M6) (hypothetical manipulation).

Let us now run through the list and check if they fulfil the manipulability theory conditions for intervention (defined in section 2.2).

According to $I N i$-iv the values of all the variables $C i$ causally connected with the effect $E$ must be fixed when an intervention is performed. This means that in order to fulfil $I N i$-iv every time we vary the value of the external decoherence $D E$ the value of the internal decoherence $D I$ should remain fixed. This is however impossible. Due to the coupling between the spin and the position, the internal and the external decoherence, and thus the variables $D I$ and $D E$ associated to them, are not independent from each other. This means that it is impossible to block one kind of decoherence in order to effectively intervene in the other one.

Thus, as a consequence of the correlation between $D I$ and $D E$, all the abovesuggested manipulations for testing this approach face a problem meeting the condition $I N-i v$.

The conclusion of this section (4.2) is the following. The decoherence-based approach provides a causal explanation of the SE-experiments (expressed in Fig. 7) but we cannot test this explanation by means of any of the manipulations presently available. Internal and external decoherences are so intricately related that every attempt to manipulate one of them will necessarily affect the other.

\section{Discussion}

In order to explain a phenomenon, interventionist explanations postulate two (or more) causes that are correlated with each other, in such a particular way, that we cannot intervene upon them separately as the manipulability theory suggests. Namely, any attempt to change the value of one cause necessarily affects the values of other causes, even when they are not on the same causal path. As a consequence, the interventionist explanations fail to meet conditions $I N-i$ or $I N-i v$ and concomitantly the criterion of explanatory depth.

The question is if from this position we shall conclude that explanations that postulate causes correlated in this particular way are not even minimally explanatory; or if we shall rather conclude that it is simply impossible to test those explanations by means of the manipulability theory. The interventionist's explanations are unable to provide interventions under which the postulated causes remain invariant. Are explanations in this situation ought to be classified as "shallow explanations"? Or are "shallow explanations" those whose causal relationships are non-invariant under interventions? If an explanation involves a causal relationship that is invariant only under one single intervention, is it above the threshold?

Following the manipulability theory very strictly one may simply conclude that there is no way of testing this specific causal relationship. Or one may conclude that 
the explanations of the irreversible behaviour of the spin-echo system are not explanatorily deep. This conclusion would be welcomed by critics of interventionism. They could argue, for example, that interventionist explanations are unable to show adequate interventions of the spin-echo system since interventionism approaches the irreversibility problem the wrong way. And, as the case of the multi-pulse sequences shows, interventionists still need to solve the difficulty of defining the system and the environment unambiguously.

Alternatively, one may adopt a more permissive attitude about the conditions INi-iv. Something like the following could then be argued: An intervention should ideally fulfil the conditions INi-iv; but manipulations that fail to meet one condition, should not be dismissed straight away. In cases like that, one should rather try to solve the problem by identifying the appropriate causal variables and interventions.

In accordance with this flexible attitude, one could redefine the variables and the manipulations, or even re-interpret or relax the conditions $I N i$-iv. Let me illustrate this by means of the following example (taken from Woodward 2003, 323). Suppose that a biologist studies the growth of a plant and finds a causal relationship between the height of the plant and the amount of water and fertilizer that the plant receives. Let $X_{1}=$ the amount of water, $X_{2}$ the amount of fertilizer and $Y=$ the height of a plant. According to the biologist the functional relation $Y=a X_{1}+b X_{2}$ and Fig. 8 (below) represent the causal relation between these variables.

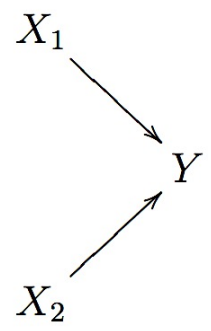

Figure 8

And suppose that the only way of adding fertilizer to the plant implies adding some water too, because most of the available fertilizers are diluted in water; and even if we get some solid fertilizer and place it inside the flowerpot, the plant does not absorb it unless we add some water. This means that manipulations of the variable $X_{2}$ unavoidably yield changes in the value of $X_{1}$ in violation of requirement $I N-i v$. Therefore, there are no possible testing interventions upon the variable $X 2$.

The biologist, however, insists that the fertilizer is a cause of the plant's growth and argues that, even though it is not possible to intervene upon $X 2$, the causal relation between $\mathrm{X}_{2}$ and $Y$ is genuine because it is invariant under a range of "controlled changes" in both $X_{1}$ and $X_{2}$. By measuring the amounts of water and fertilizer added to the plant, it is possible to know both the change in the variable $X_{1}$ (call it $\Delta X_{1}$ ) and the change in the variable $X_{2}$ (call it $\Delta X_{2}$ ). And the biologist argues that the functional relation is invariant under manipulation because the total change in the variable $Y$ is 
exactly what the functional relation says it is, namely $\Delta Y=a \Delta X_{1}+b \Delta X_{2}$ (see Woodward 2003, 324).

Someone adopting the flexible attitude would say that an explanation of the plant's growth appealing to this functional relationship fulfils the criterion of explanatory depth-or a flexible version of criterion 1 that admits invariance under the changes explained above, even though they are not interventions stricto sensu.

If, by an analogous argument, we flexibly applied conditions $I N-i$ and $I N-i v$ to both the classical and the quantum interventionist explanations of the spin-echo experiments, they would be evaluated as possessing some explanatory depth. The causal relationships postulated by interventionists are as if the variables $X_{1}$ and $X_{2}$ in the plant's example were intertwined in such a way that changing the value of $X 1$ leads to changes in the value of $X_{2}$ and vice-versa. In the classical interventionist explanation this is due to the fact that putative interventions violate requirement $I N-i$. And in the decoherence-based approach this is due to the fact that the two putative causes are in a causal structure that is precisely as the causal structure of the plant's explanation (see Fig. 8).

This means that, in order to produce an argument analogous to the biologist's argument, interventionists should provide us with quantitative predictions and functional relations of the kind $H=\mathrm{f}(E P)$ and $H=\mathrm{f}(D I, D E)$; where $H$ stands for height of the echo-signal.

They should also provide the specific intervals of values that the putative causes may take. This would enable us to assess if the functional relations correctly describe the values of the height of the echo $H$ under different changes in the putative causes (although those changes are not interventions strictly speaking). And, concomitantly, this would enable us to show to what extent the explanations meet the criterion of explanatory depth (2.3). Using these functional relations, interventionist could additionally predict relaxation times suitable to be experimentally tested.

Unfortunately, having reached this point, interventionists have not provided those functional relations. We only count on the qualitative directed graphs in order to assess the explanations. Therefore we are unable to show that the interventionist explanations fulfil the criterion (2.3) of explanatory depth. Nevertheless, the flexible attitude described in this section would allow us to argue that interventionists have offered a genuine causal account of the SE-experiments.

\section{Conclusions}

The reason why the interventionist explanations fail to meet conditions $I N-i$ or $I N-i v$, and concomitantly the criterion (2.3) of explanatory depth, is the following: In order to explain a phenomenon, interventionist explanations postulate two (or more) causes that are correlated with each other. And if the causes are correlated we cannot intervene upon them in the way that the manipulability theory considers valid.

Rejecting explanations that postulate correlated causes leads to some kind of scepticism and this, in my view, is a weakness of the manipulability theory. Suppose an explanation has already been evaluated as deep because it is invariant under several interventions. This explanation is always at risk of becoming unsatisfactory if a new 
causal factor, correlated with the originally proposed cause, is discovered. Even if the new factor is genuine and provides more information for controlling the explained event, it will automatically render invalid all the interventions that were previously considered appropriate. Therefore, the explanation will fail to meet the criterion (2.3) of explanatory depth, and hence will lose its depth. A sceptic would thus claim that we should always shed doubts on the depth of the explanation because we never know whether there is an unknown correlated cause.

In other words, diagnostics of explanations obtained through the application of the manipulability theory are as lucky as scientific theories. They may be amended or abandoned. This would be acceptable if the arrival of new causal information would produce stronger and deeper explanations. However, in diagnostics obtained via the manipulability theory, the new causal information far from strengthening the explanations, seems to render vulnerable explanations that were already considered to be explanatorily deep.

Fortunately, as we have seen in section 5, adopting a flexible attitude towards conditions INi-iv, in very particular cases, may be useful to assess the import of causal relationships despite the fact that those manipulations are not interventions in the strict sense. And the interventionist explanations of the SE-experiments are one of these particular cases. We have discovered that, under this flexible perspective, interventionist could gain explanatory depth by providing functional relations and predicting specific relaxation times.

I would finally like to stress that the conclusions of this article are critical but constructive. It is suggested that clarification of specific aspects of the manipulability theory would be illuminating. However, it is never suggested the manipulability theory has not served us to analyze the explanations of the SE-experiments. On the contrary, the manipulability theory has helped us to visualize the advantages and disadvantages of those explanations from a new perspective, and has provided us with some specific proposals to improve the interventionist explanations of irreversibility. Namely, interventionist should provide functional relationships and predictions of the relaxation times for the SE-experiments.

\section{REFERENCES}

Bergmann, Peter and Joel Lebowitz. 1955. New Approach to Nonequilibrium Processes. Physical Review, 99(2), 578-587.

Bloembergen, Nicolaas, Edward Mills Purcell, and Robert Pound. 1984. Relaxation Effects in Nuclear Magnetic Resonance Absorption. Physical Review, 73(7), 679-712.

Carr H.Y., and E.M. Purcell. 1954. Effects of diffusion on free precession in nuclear magnetic resonance experiments. Physical Review, 94(3), 630-638.

Hahn, Erwin. 1950. Nuclear Induction Due to Free Larmor Precession. Physical Review 77(2), 297-298.

—. 1953. Free Nuclear Induction. Physics Today, 6(4), 4-9.

Hemmo, Meir and Orly Shenker. 2003. Quantum Decoherence and the Approach to Equilibrium. Philosophy of Science, 70 (2), 330-358.

-. 2005. Quantum decoherence and the approach to equilibrium (II). Studies in History and Pbilosophy of Modern Physics, 36, 626-648.

Martin, Daniel and Paul Hughes. 2006. Spin Echo NMR. Laboratory report, School of Physics and Astronomy, The University of Manchester. 
Meiboom, S. and D. Gill. 1958. Modified Spin-Echo Method for Measuring Nuclear Relaxation Times. Review of Scientific Instruments 29(8), 688-691.

Nyenhuis, J.A and O.P Yee. 1994. Simulation of nuclear magnetic resonance spin echoes: Influence of magnetic field inhomogeneities. Journal of Applied Physics 76(10), 6909-6911.

Ridderbos. T.M, and M. L. G. Redhead. 1998. The Spin-Echo Experiments and the Second Law of Thermodynamics. Foundations of Physics, 28(8), 1237-1270.

Shenker, Orly. 2001. Interventionism in statistical mechanics: Some philosophical remarks. Philosophy of Science Archive of the University of Pittsburgh. http://philsci-archive.pitt.edu/151/.

Suárez, Mauricio and Iñaki San Pedro. 2011. Causal Markov, Robustness and the Quantum Correlations. In M. Suárez (ed.), Probabilities, Causes and Propensities in Physics (173-193). New York: Springer.

Stejskal E. O. and J. E. Tanner. 1965. Spin Diffusion Measurements: Spin Echoes in the Presence of a Time Dependent Field Gradient. Journal of Chemical Physics, 42, 228-293.

Uffink, Jos. 2004. Bluff your way trough the Second Law of Thermodynamics. Studies in History and Philosophy of Modern Physics, 32(3), 305-394.

Uhring, Gotz S. 2007. Keeping a Quantum Bit Alive by Optimized pi-Pulse Sequences. Physical Review Letters, 98, 100504-1 to 100504-4.

—. 2009. Concatenated Control Sequences Based on Optimized Dynamic Decoupling. Physical Review Letters 102, 120502-1 to 120502-4.

Woodward, James. 2003. Making Things Happen: A Theory of Causal Explanation. Oxford Studies in the Philosophy of Science: Oxford University Press.

- 2008. Causation and Manipulability. The Stanford Encyclopedia of Philosophy, Edward N. Zalta (ed.) http://plato.stanford.edu/archives/sum2013/entries/causation-mani/ .

Fernanda Samaniego After she graduated in Physics and completed two master tittles in Philosophy of Science (at the National Autonomos University of Mexico, UNAM) and in Philosohy and History of Science (at the London School of Economics), she obtained her PhD with European Mention at the Complutense University of Madrid. She is currently holding a postdoctoral fellowship at the Institute of Philosophical Research, UNAM.

Address: Instituto de Investigaciones Filosóficas, UNAM, Circuito Mtro. Mario de la Cueva s/n, Ciudad Universitaria, C.P. 04510, Coyoacán, México, D.F. E-mail: fernanda.samaniego@gmail.com 